Gesnerus 63 (2006) 73-89

\title{
Délires de métamorphose
}

Jackie Pigeaud

\section{Summary}

Two of the most eminent 17th-century doctors, Sydenham and Willis, were led by their observations of melancholy people's relations with their bodies to elaborate a conception of the internal man. The present article uses their studies of hypochondria as a male form of hysteria to show their similarities and differences. Both doctors attempted to explain how strange ideas produce true sensations; but while Sydenham saves the individual's unity by supposing an internal double (a solution later developed by others, Cabanis in particular), Willis insisted on the difference between the metaphor enacted by the melancholic for himself and his healthy state. They represent two different ways of continuing the inheritance of Aristotelian mimesis.

Keywords: melancholy; Thomas Sydenham (1624-1689); Thomas Willis (1621-1675); hypochondria; imagination; lycanthropy; Athanasius Kircher (1602-1680)

\section{Résumé}

L'observation des relations que les mélancoliques entretiennent avec leur corps a conduit deux des plus éminents médecins du XVII ${ }^{\mathrm{e}}$ siècle, Sydenham et Willis, à élaborer un concept de l'homme intérieur. Cet article s'appuie sur leurs études de l'hypocondrie comme forme masculine de l'hystérie pour montrer leurs points de convergence et de divergence. L'un et l'autre cherchent à expliquer comment des idées bizarres produisent des sensations vraies. Mais alors que Sydenham sauve l'unité de l'individu en posant un double intérieur - solution qui aura les développements que l'on sait, en particulier avec Cabanis -, Willis va insister sur la distance entre la métaphore

Prof. Jackie Pigeaud (Université de Nantes, Institut Universitaire de France), 2, chemin de la Berthelotière, F-44700 Orvault (jackie.pigeaud@wanadoo.fr). 
de lui-même que le mélancolique met en scène et ce qu'il est dans l'état sain. Deux manières différentes de reprendre l'héritage de la mimésis aristotélicienne.

Quand on parle de métamorphoses, on ne risque pas grand chose à dire qu'Ovide est le maître absolu. Cela concerne aussi un peu la psychopathologie. La question de l'hypocondrie, maladie un peu oubliée, banalisée, méprisée comme assez peu glorieuse finalement, disons l'hypocondrie peut être abordée avec le dessein, un peu surprenant d'abord, de l'interroger sur sa qualité poétique. Stricto sensu, c'est la maladie qui affecte les hypocondres, c'est-à-dire les parties musculeuses situées entre le condre ${ }^{1}$ et le nombril. Mais on sait que l'hypocondrie est devenue une sorte de rivale de la mélancolie, inventée comme telle, par le médecin Dioclès de Caryste ${ }^{2}$, au $\mathrm{IV}^{\mathrm{e}}$ siècle avant J.-C. Il s'agit ici de saisir l'hypocondrie à un moment où elle se constitue de manière nouvelle et forte, au XVII ${ }^{\mathrm{e}}$ siècle avec Sydenham (1624-1689) et Willis (1621-1675).

\section{Thomas Sydenham (1624-1689)}

Pour Sydenham, grand protagoniste de l'histoire des maladies, force est de constater qu'il existe une maladie dont on ne peut faire l'histoire, une maladie anhistorique, on pourrait dire une maladie anecdotique, faite d'histoires, et celles-là bien singulières. Commençons par la «lettre à Cole». Dans une lettre qu'il avait adressée à Sydenham, après avoir demandé des indications sur la petite vérole, Cole ajoute:

J'ai appris aussi par la même personne (un certain Kendrik) que vous aviez fait des observations rares sur les affections qu'on appelle hystériques (affectus hystericos dictas), ces maladies qui ont été de tout temps inexplicables aux plus savants médecins et qui ne résistent que trop aux remèdes $[\ldots]{ }^{4}$

1 Cartilage qui termine le sternum.

2 Médecin influencé par Aristote, selon Jaeger 1938.

3 L'œuvre de Sydenham a d'abord été écrite en anglais, puis remise à Mapletoft et Havers, qui la traduisirent en latin. «Ce furent ces traductions qui furent livrées à la postérité; les originaux furent détruits», selon Dechambre, dans le Dictionnaire Encyclopédique des Sciences médicales. L'œuvre paraît, traduite en anglais, en 1742, avec des notes de Swan. En français, c'est le médecin Jault qui la traduit à partir de l'anglais. Il faut prendre garde aux lacunes de cette traduction et toujours recourir à la version latine. Les lacunes de Jault sont évidemment celles de Swan, le traducteur anglais ( $1^{\text {re }}$ éd. 1742).

4 Jault 1776, 437, traduction légèrement modifiée. Sydenham 1716, t. I, 384. 
Sydenham répond qu'il considère la maladie hystérique comme la plus fréquente après la fièvre ${ }^{5}$. C'est une maladie très difficile, qui exige du médecin astuce et sagacité. Il s'agit de démasquer la vraie maladie (l'hystérie) sous la fausse apparence:

L'affection hystérique est fréquente; elle se montre sous une infinité de formes, et elle imite presque toutes les maladies dont les malheureux mortels sont écrasés; car, dans quelque partie du corps qu'elle se rencontre, elle produit aussitôt les symptômes qui sont propres à cette partie. Et si le médecin n'a pas beaucoup de sagacité et d'expérience, il se trompera aisément, et attribuera à une maladie essentielle et propre à telle ou telle partie, des symptômes qui dépendent uniquement de l'affection hystérique. ${ }^{6}$

Au passage il constate une abondance d'urine claire comme l'eau de roche (autant chez «les femmes hystériques que chez les hommes hypochondriaques» $\left.{ }^{7}\right)$. C'est qu'il y a une identité entre ces deux formes essentielles que prend la maladie selon les sexes: l'hystérie pour les femmes, et ce qu'il est convenu d'appeler l'hypocondrie pour les hommes. Galien avait eu l'idée qu'il y avait chez les hommes l'analogue de l'hystérie ${ }^{8}$. Chez les femmes, la maladie était due à l'affection de l'utérus, souvent à l'absence de rapports. Pour les hommes, la rétention de sperme pouvait aboutir à des symptômes semblables. Mais, de manière évidente, puisque l'utérus est le siège de la maladie hystérique, on ne pouvait parler vraiment d'une hystérie masculine.

\section{Comment nommer: la désignation ${ }^{9}$}

Tant les femmes hystériques que les hommes hypochondriaques ont des problèmes digestifs, écrit Sydenham.

J'ajoute que presque toutes les femmes hystériques se plaignent d'un grand abattement des esprits (animaux) ou d'un dépôt, pour parler leur langage, accusant la région du poumon, toutes les fois qu'elles veulent désigner le lieu où se fait sentir la contraction ou le dépôt des esprits. $^{10}$

5 Il répond volontiers à Cole (Sydenham 1716, 385, passage non traduit par Jault) en se plaignant de la goutte qui le tourmente et de l'âge qui vient. La lettre trompera «l'ennui des brumes de la nuit $[\ldots] »$.

6 Traduction Jault modifiée, Jault 1776, 476. Voir aussi: «Les femmes hystériques passent du rire aux larmes sans prophasis manifeste.» Sydenham 1716, 408.

7 «J'ai quelquefois observé dans les hommes que, peu de temps après avoir rendu une urine de couleur citrine, s'ils venaient à être agités tout-à-coup d'une passion violente, ils rendaient sur le champ une grande quantité et pendant longtemps une urine claire, et qu'ils se trouvaient mal jusqu'à ce que l'urine ait repris sa couleur naturelle. Car alors le paroxysme se terminait», Sydenham 1716, 411; Jault 1776, 480.

8 Galien, De locis affectis, VI, 5, avait constaté une analogie entre les troubles de la femme et ceux de l'homme condamné à une rétention de sperme. Galien 1856, t. 2,687ss.

9 Sydenham 1716, 411; Jault 1776, 480.

10 Non traduit par Jault. J'ai longuement parlé ailleurs de cette question de la désignation: cf. Pigeaud 1999 et 2001. 
Ces malades souffrent plus de l'âme que du corps ${ }^{11}$. Certes ils souffrent horriblement du corps. Sydenham décrit cette souffrance en termes violents pour faire valoir qu'elle n'est rien à côté du désespoir tout à fait incurable qui les tient $^{12}$. Ils se mettent en colère si on leur donne fût-ce le plus petit espoir de guérison:

Ils s'abandonnent pour le moindre sujet, et même sans sujet à la crainte, à la colère, à la jalousie, aux soupçons [...] et ils se tourmentent eux-mêmes. Ils ne peuvent souffrir la joie [...]. Ils ne gardent aucune médiocrité, ils ne sont constants que dans leur légèreté [...] ils changent aussitôt de dessein, et entreprennent tout le contraire sans néanmoins l'achever [...] ils sont dans des inquiétudes continuelles. ${ }^{13}$

Alors manque, dans la traduction de Jault (qui suit l'anglais de Swan), tout le développement culturel, pourtant capital:

Et ce que dit l'Orateur romain des superstitieux s'accorde admirablement avec nos mélancoliques. ${ }^{14}$ Le sommeil semble le refuge de tous les maux et de tous les soucis, mais de luimême naissent le plus de troubles et de craintes. ${ }^{15}$

Par exemple, ils voient des morts ... Ils sont déchirés dans leur âme comme dans leur corps:

Ce n'est pas seulement à des maniaques et furieux que ce que je dis arrive, mais aussi à des hommes très sages et sensés, et qui ont une pénétration et une sagacité extraordinaire. C'est donc à grande raison qu'Aristote a observé que les mélancoliques l'emportent sur tous les autres par leur génie. ${ }^{16}$

\section{De Protée à l'homme intérieur}

Je ne finirais point si j'entreprenais de rapporter ici tous les symptômes de l'affection hystérique, tant ils sont différents, et même contraires les uns aux autres. Cette maladie est un Protée qui prend une infinité de formes différentes. C'est un caméléon qui varie sans fin ses couleurs. Aussi Démocrite me paraît avoir eu raison d'assurer, dans sa lettre à Hippocrate ${ }^{17}$, que l'affection hystérique était la source d'une infinité de maux, quoiqu'il se trompât en assignant la matrice pour causes de cette maladie $[\ldots]^{18}$

11 Sydenham 1716, 411.

12 «Desperatio plane insanabilis de hujusce morbi natura (fit) [...].» Sydenham 1716, 412.

13 Jault 1776, 481; Sydenham 1716, 412.

14 Il s'agit là d'une citation de Cicéron, De divinatione II, 150.

15 En somme, c'est la parodie de la situation tragique, celle que j'appelle «moderne», l'impossibilité de mourir, cf. Longin 1993. Il faut citer le latin: «Quodque de superstitiosis afferit Orator Romanus, nostris hisce melancholicis pulchre quadrat: «Perfugium videtur omnium laborum et sollicitudinum esse somnus, at ex eo ipso plurimae curae metusque nascuntur $>. »$

16 Sydenham cite sans aucun doute Cicéron, De divinatione I, 38, 81, par qui il doit connaître le Problème XXX. Dewhurst rapporte l'histoire suivante: «His 〈chamber fellow〉 at All Souls, Dr. Thomas Millington, mentions to Lord Pembroke [...] that Sydenham had entirely forgotten his Latin on the return from the Civil War, but he recovered it reading Cicero», Dewhurst 1966, 72 .

17 Il s'agit de sa lettre sur la Nature de l'homme, IX L 396.

$18 \ll[\ldots]$ tam diversa atque ab invicem contraria specie variantia, quam ne Proteus lusit unquam, nec coloratus spectatur Chamaeleon. Proinde recte mihi videtur rationem subducisse Demo- 
Aucune règle; aucun type uniforme; aucune histoire possible ${ }^{19}$. L'origine est en fait l'ataxie; le grand désordre des esprits animaux. La cause en est la trop grande faiblesse de ces esprits animaux ${ }^{20}$.

C'est ici que se trouve le passage essentiel, que j'ai commenté souvent, définissant l'homme intérieur. Jault ne traduit malheureusement pas ce passage. Sans doute l'estime-t-il trop philosophique pour un médecin ${ }^{21}$.

Car de même en effet qu'il existe un homme extérieur, qu'on peut voir, formé de l'assemblage des parties qui se montrent aux sens, de même, sans nul doute, il existe aussi un homme intérieur, constitué par l'enchaînement nécessaire et, pourrait-on dire, la constitution ${ }^{22}$ des esprits animaux, et que seul peut contempler la lumière de la raison. Or celui-ci, conjoint de manière très intime au tempérament du corps, et, pour ainsi dire, ne faisant qu'un avec lui, voit son état se dégrader de manière d'autant plus maladive et facile que la robustesse des principes constituants, que la Nature nous octroie, est plus grande ou plus faible. ${ }^{23}$

Mettons de côté les esprits animaux, dont l'origine et la source est le sang ${ }^{24}$. Pour rendre compte de l'unité de l'individu, dans cette maladie protéiforme qu'est l'hypocondrie ou l'hystérie, finalement, comme on l'a vu, formes de la mélancolie, il faut supposer l'existence d'un double intérieur, intimement lié à l'homme extérieur, mais tourné, si l'on peut dire, vers l'intérieur, et vivant spontanément, sans intermédiaire, les fluctuations physiologiques. Peut-être

critus (quamlibet in morbi causa hallucinaretur), ubi in Epistola ad Hippocratem misa dierte asserit: 〈Sexcentarum aerumnarum, innumerarumque calamitatum authorem esse uterum〉», Sydenham 1716, 408.

19 Jault 1776, 481; Sydenham 1716, 412.

20 Sydenham 1716, 413.

21 «Quemadmodum enim Homo quidam exterior conspicitur ex partibus sensui obviis compaginatus, ita procul dubio \& interior est quidam Homo, et debita spirituum serie, et quasi Fabrica, constans, solo rationis lumine contemplandus. Hic vero cum temperie corporis intimius conjunctus \& quasi unitus, tanto aegrius faciliusve de statu suo dejicitur, quanto major est minorve ea, quam a natura sortimur, Principiorum constituentium firmitas», Sydenham 1726, 395. Voici la traduction de Dewhurst: «Just in the same way that a certain external man can be perceived, made of the combination of parts that appear to senses, so, without any doubt, there is, too, an internal man, made of the necessary connection, and, so to speak, the workshop of the animal spirits, and which can be only observed by the light of Reason. This internal man, connected more intimately with the temperament of the body, and, so to speak, being one with it, is thrown from its proper state, all the more or less gravely and easily, because the firmness of the constitutive principles that Nature delivers, is more or less valuable», Dewhurst 1966, 133s.

22 Quel est ici le sens de fabrica? Atelier, comme je l'ai traduit ailleurs? Il vaudrait mieux traduire par l'organisation, la structure. Plus loin, Sydenham parle de «compages animi, si fas est ita dicere [...]», Sydenham 1716,417. On ne saurait oublier, bien entendu, le titre de Vésale, De humani corporis fabrica (Bâle 1543).

23 On pourra apprécier cette traduction jointe au livre de Robert Whytt: «Outre l'homme que l'on peut appeler extérieur, \& qui est composé de parties qui tombent sous les sens, il y a un homme intérieur, formé du système des esprits animaux, \& qui ne se peut voir que par les yeux de l'esprit. Ce dernier, étroitement joint, \& pour ainsi dire, uni avec la constitution corporelle, est plus ou moins dérangé de son état, selon que les principes qui forment la machine ont reçu plus ou moins de fermeté de la nature [...]», Whytt 1777, 403.

24 Sydenham 1716, 418; Jault 1776, 490. 
la distinction entre l'homo interior et l'homo exterior est-elle augustinienne dans son origine ${ }^{25}$. Mais l'opposition, chez Sydenham, n'a aucune valeur morale. Ce double permet d'éviter la question de la médiation, si difficile à expliquer, si l'on est dualiste, entre l'âme et le corps, et laisse libre une interprétation dualiste - si l'on insiste sur la composition des esprits animaux - et une interprétation parfaitement moniste, celle d'un recto/verso, si l'on peut dire, ou d'un moderne signifiant/signifié.

Cette distinction arrive, semble-t-il à point, si l'on en croit Cabanis (17571808). Ce dernier recueille précieusement le texte de l'Hippocrate anglais, comme on l'appelait, et le paraphrase:

Ainsi donc, selon l'expression de Sydenham, il y a dans l'homme un autre homme intérieur, doué des mêmes facultés, des mêmes affections, susceptible de toutes les déterminations analogues aux phénomènes extérieurs, ou plutôt dont les faits apparents de la vie ne font que manifester au-dehors les dispositions secrètes, et représenter en quelque sorte les opérations. ${ }^{26}$

Et Cabanis conclut: «Cet homme intérieur, c'est l'organe cérébral [...].» Mais cela n'est point de Sydenham.

Revenons à Protée. «La disposition au phantasme, n'est jamais aussi commune que dans l'hypochondrie» ${ }^{27}$, écrit Kant en 1764, qui décrit cette dernière comme une maladie où le malade se trompe, s'hallucine sur son propre état intérieur (âme ou corps), où il souffre d'un mal qui,

quel que soit le siège principal, parcourt le tissu nerveux du corps, déploie un souffle mélancolique $^{28}$, autour du siège de l'âme, de sorte qu'il croit avoir toutes les maladies dont il entend simplement parler. Ce sont là ses hallucinations. ${ }^{29}$

S'il ressemble beaucoup à l'homme dérangé, il n'est pas pourtant en situation d'urgence. «Le mal n'a pas de racines profondes et se dissipe généralement, en ce qui concerne l'esprit, soit spontanément, soit grâce à un médicament.» ${ }^{30}$ L'hypocondrie est faite d'éléments difficiles à ramener sous un même concept. Du point de vue qui nous intéresse, celui du phantasieren, elle intrigue fortement, car elle est maladie du mensonge, de la tromperie, de l'illusion. Elle trompe le malade comme le médecin ${ }^{31}$.

25 Cf. par exemple l'esquisse de la formation de la notion de conscience dans Pigeaud 1995.

26 Cabanis 1956, 209.

27 Kant 1990, 63s. (traduction modifiée).

28 Le souffle est à prendre au sens littéral.

29 Kant 1990, 64 (traduction modifiée).

30 Kant 1990, 65.

31 «Et nisi Medicus tam sagaci quadam sollertia [...] quam in ei arte peritia valeat, fraus ei fiet, atque ista symptomata a morbo aliquo essentiali [...] non vero ab affectione hysterica, pendere arbitratur», Sydenham 1716 (Lettre à Cole), 408. 
La première thèse de l'existence de troubles hystériques masculins remonte sans doute à Carolus Piso ou Charles Le Pois (1563-1633) ${ }^{32}$. Ce médecin, réédité au XVIII ${ }^{\mathrm{e}}$ siècle par Boerhaave, est couvert par lui d'éloges, bien mérités selon Calmeil ${ }^{33}$ :

On a agité cent fois la question de savoir si les hommes peuvent, aussi bien que les femmes, être atteints de convulsions hystériques; cette question est toute résolue par la théorie de Lepois [Piso]. Puisque le siège des désordres [...] réside dans les hémisphères cérébraux et non dans l'appareil de la génération, il n'y a pas de raison pour que l'hystérie épargne absolument les hommes, et l'observation clinique prouve qu'effectivement ils sont quelquefois exposés à cet état maladif. Ch. Pison [Piso] a cherché aussi à constater sur des cadavres des altérations $[\ldots] \cdot{ }^{34}$

Ayant constaté chez lui des troubles analogues à ceux d'une jeune fille migraineuse, il écrit que le seul siège que l'on puisse accuser est la tête et non le $\operatorname{sexe}^{35}$. On pourrait dire les choses autrement. Il existe une maladie identique qui frappe les femmes et les hommes, qu'on peut appeler, si l'on veut, hystérie ou hypocondrie.

\section{Thomas Willis (1621-1675)}

On néglige trop ce grand contemporain de Sydenham qu'est Willis. Pour la mélancolie dans les œuvres de Thomas Willis, nous avons deux sources: les Willis Oxford Lectures, publiées par Kenneth Dewhurst - ce sont les notes prises par Locke (1632-1704) et Richard Lower (1631-1691) -, et le texte latin de Willis lui-même ${ }^{36}$.

Les troubles de l'imagination reposent sur le désordre des esprits animaux, dont la nature présente une analogie avec les esprits extraits par procédés chimiques. Ce sont les esprits inflammables, constitués d'esprits du genre des esprits de vin. Quand on trouve ce genre d'esprit dans des fièvres ou des excès de boisson, ils deviennent agiles, sans repos, et rapidement inflammables. Ils disparaissent vite et la diathèse ne dure pas longtemps. Ce sont les esprits composés d'esprit et de sel volatiles, qui ne sont pas inflammables, mais subtils et agiles, comme des esprits distillés de sang $[\ldots]^{37}$

32 Charles Le Pois (1563-1633). Voir la réédition de Piso au XVIII ${ }^{\mathrm{e}}$ siècle: Piso 1733,181. Piso en effet écrit qu'il a ressenti des symptômes semblables à ceux d'une jeune femme; comment alors dans le sexe féminin accuser l'utérus et non la tête?

33 Louis Florentin Calmeil (1798-1895), médecin aliéniste et élève d'Esquirol.

34 Calmeil 1845, t. I, 377.

35 «Que si ces maux de tête, que je partage avec des femmes, sont les effets d'une seule et même cause, et si, à coup sûr ils ne peuvent être attribués chez moi à l'utérus, à moins d'accepter le ridicule et l'absurde, pourquoi accuser chez la femme l'utérus et non la tête?» Charles Le Pois $1733,180$.

36 Willis 1676.

37 Willis 1676, 239. 
Suit une comparaison avec le microscope; les rayons les plus concentrés agrandissent les petits objets et les rendent terrifiants pour les yeux ${ }^{38}$. Ailleurs Willis utilise l'analogie avec le verre déformant qui ne montre qu'une partie des choses. Et voici que Willis, lui aussi, propose un homme intérieur, mais avec une définition beaucoup plus précise que celle de Sydenham:

Cela semble consister en un tissu d'esprits animaux et constitue une sorte d'homme éthéré, constitué des atomes les plus subtils, et qui est coextensif à notre corps. De plus ce génie qui nous est propre quelquefois se répand en dehors de notre corps, comme cela arrive dans la joie, l'impatience, le courage; d'autres fois il se rétracte au point de n'être plus coextensif avec notre corps, et il change à l'occasion de forme, subissant des métamorphoses, pourrait-on dire, et s'emparant de formes variées. ${ }^{39}$

Dans la joie, par exemple, la systasis des esprits animaux se précipite violemment («hurls itself»), comme hors du corps, «and as in the proverb, men are besides themselves $[\ldots] »^{40}$. Cela est causé par «le désir d'objets externes ou internes $[\ldots]$ ». Les malades sont «à côté d'eux-mêmes» ${ }^{41}$. C'est en somme la coïncidence (mais physique) de soi avec soi qui fait la santé.

Dans l'amour, l'individu se dessèche au sens propre. Le génie éthéré quitte son propre corps. La raison en est que l'anima sensitiva, cet homme éthéré qui nous est coextensif, désire s'unir et s'assimiler avec l'être qu'il désire, s'étend hors de lui-même et pousse des racines dans cet être aimé d'où il tire vigueur et vie ${ }^{42}$.

Il reste à parler de la lycanthropie et de ces conditions mélancoliques associées avec l'imagination d'une métamorphose, exemples qui arrivent parfois. C'est une longue histoire. On se contentera ici de mentionner que ces conditions ne semblent pas tant consister dans la contraction ou dilatation de l'anima sensitiva, que dans le changement et la détérioration de sa forme ${ }^{43}$.

38 «So these spirits concentrated in the deepest part of the brain exaggerate the phantasy giving these things a greater importance than they really have», Dewhurst 1980, 123. Sur le verre déformant et qui ne montre qu'une partie des choses, voir Willis 1676, 239, et 241 pour le modèle du microscope.

39 «This seems to consist of a contexture of animal spirits and is a sort of aetherial man made up of the most subtle atoms being coextensive with our body. Furthermore this genius of ours sometimes expands beyond our body, as in joy, eagerness and boldness; other times it contracts so as not to be coextensive with our body, and occasionally changes its shape, undergoing metamorphoses, as it were, and taking on various forms», Dewhurst 1980, 125.

40 Dewhurst 1980, 126.

41 Dewhurst 1980, 126.

42 Dewhurst 1980, 128s.

43 Dewhurst 1980, 129. Willis esprit spéculatif? Certes. Mais, comme le souligne Dewhurst, ces spéculations sont faites sur le fond d'un arrière-plan scientifique qui implique des recherches dans les domaines de la iatrochimie, de l'anatomie comparée, de la chirurgie expérimentale, de la pathologie, de l'expérimentation et des dissections sur les animaux. 


\section{La lycanthropie}

Est donc venu, comme naturellement, le nom de la lycanthropie ${ }^{44}$.

L'Antiquité faiseuse de mythes n'a à peu près imaginé aucune métamorphose d'homme telle qu'on ne puisse trouver quelques-uns qui l'aient acceptée vraiment en ce qui les concerne,

écrit Willis.

Les uns se croient des chiens ou des loups, et imitent leurs mœurs et leur aspect, par l'aboiement ou le hululement; d'autres croient être morts, demandant qu'on les mettent aussitôt au tombeau; d'autres s'imaginent avec un corps de verre $[\ldots] .{ }^{45}$

Il ne faut pas laisser les mélancoliques seuls:

Si en effet ils se laissent aller à leurs phantasmes et leurs spéculations aériennes et s'y tiennent longtemps, l'âme à l'intérieur se déposera et, se séparant du corps, et subissant une métamorphose, revêt une nouvelle forme, et souvent étrangère aux mours humaines. ${ }^{46}$

Le mélancolique peut sortir de l'humanité.Il se métamorphose. Willis revient encore sur ces «métamorphoses imaginaires des mélancoliques» ${ }^{47}$, décrivant quelques-unes des métamorphoses de leurs fortunes ou de leurs corps. L'un s'imagine prince, l'autre mendiant et agissent comme tels; l'un croit avoir un corps de verre, un autre croit être un chien ou un loup ${ }^{48}$ ou un autre monstre; après que l'âme corporelle affectée d'une mélancolie chronique,l'esprit étant aveuglé, s'est séparée complètement d'elle-même et du corps, elle prend un aspect nouveau ou une condition nouvelle, et, autant qu'elle le peut, elle l'assume vraiment.

Nous avons vu l'analogie, chez Willis, entre processus chimiques et mélancolie. La mélancolie, comme la manie, s'illustre très bien par l'analogie avec les liqueurs chimiques:

Alors est mis en mouvement un bouillonnement perpétuel des pensées, dont le cerveau est sans cesse occupé, au point que les mélancoliques ont nuit et jour l'imagination [phantasiam] perturbée $[\ldots]^{49}$

On comprend que la lycanthropie soit comme la manifestation extrême de ces changements de formes. Freind ${ }^{50}(1675-1728)$ cite Oribase, le médecin de l'empereur Julien au $\mathrm{IV}^{\mathrm{e}}$ siècle, comme le premier à avoir décrit cette maladie, pour lui une sorte de la mélancolie.

44 Melancholia zooanthropia chez Lycaon d'Aetius. Les autres noms de la mélancolie sont: galeanthropia, lycanthropia, dans la classification de Sauvages.

45 Willis $1676,238$.

46 Willis 1676, 246.

47 Willis 1676, 254.

48 En italique dans le texte.

49 Willis $1676,254$.

50 Freind 1728,8 . 
C'est une espèce de mélancolie ou de rage, qu'il appelle lycanthropie ou lycanthrope. Les personnes affligées de cette maladie sortent la nuit de leur maison, \& imitent en tout les loups, ils errent parmi les sépulcres jusqu'à la pointe du jour. Vous pouvez les connaître à ces symptômes: ils ont la mine pâle, les yeux chargés, creux, secs [...]; leur langue est sèche et brûlante, la salive tarie [...]. Aetius appelle cette maladie cynanthropie, aussi bien que lycanthropie..$^{51}$

Black, dans sa courte mais précieuse histoire ${ }^{52}$, remarque qu'Oribase traîne avec lui tous les lieux communs de la manie, mais qu'il en ajoute une, originale celle-ci, la lycanthropie; et il évoque à ce propos Platon. Nous y reviendrons plus loin.

$\mathrm{Au} \mathrm{VII}{ }^{\mathrm{e}}$ siècle après J.-C., le médecin Paul d'Egine ${ }^{53}$ donne de cette maladie la même relation qu'Oribase. Evidemment il faut penser à la métamorphose d'Ovide, ce qu'on n'a pas manqué de faire. Par exemple Jean Wier, le médecin démonologue (1515-1588):

Cette maladie qui fait que les hommes pensent estre loups, est nommée par les Grecs Lycanthropie: quelques-uns la nomment mélancholie louvière, selon Marcel ${ }^{54}$; item folie louvière; quelques-uns la nomment aussi Lycaonie, \& les autres Cynanthropie. Ceux qui sont malades de cette maladie, pensent être transformés en loups, ou en chiens: ce qui leur advient par les fumées de la mélancholie ou cholere noire [...].

Il est vraysemblable que ce vice naturel \& perte de l'esprit humain a donné occasion à la fable escrite par Ovide, de Lycaon roy d'Arcadie, lequel comme il écrit, fut à raison de ses mesfaicts changé en loup, par Jupiter. Cependant il adjouste les accidents \& les signes de la lycanthropie, ainsi que souventes fois les foles inventions des poètes ont accoutumé d'estre apuiées sur les causes naturelles: il écrit doncques: «Il s'enfuit estonné et trouvant le silence / Dans les champs esgarez, qu'il quiert pour demourance / Il hurle, \& vainement il s'efforce à parler.» Avicène a remarqué en ses livres que plusieurs tourmentéz par cette cholère noire ont pensé être lions, ou diables, ou oyseaux [...]. D'autres se sont crus transformés en ours. ${ }^{55}$

\section{Si l'on ouvre le Dictionnaire de Robert James traduit par Diderot à l'article lycanthropie ${ }^{56}$, on retrouvera Ovide:}

Terrifié il fuit et ayant trouvé le silence de la campagne il hurle et c'est en vain qu'il essaie de parler; sa bouche rassemble la rage qui sort de lui; il tourne contre les troupeaux sa passion habituelle du meurtre, et maintenant encore il se plaît dans le sang. Ses vêtements se changent en poils, ses bras en pattes; il est devenu loup et conserve les traces de son ancienne

51 «Les malades pris de lycanthropie sortent de nuit, en imitant tout à fait les loups, et séjournent jusqu'au jour autour des sépulcres», Oribase 1851, 414s.

52 «On pourrait présumer que c'est la même folie ou peut-être la même fourberie qu'on connaît aujourd'hui sous le nom de loup garou», Black 1798,147, note 1.

53 Médecin du VII ${ }^{\mathrm{e}}$ siècle après J.-C.

54 Soit le médecin latin Marcellus.

55 Wier 1885, t. I, 596. Jean Wier parle d'abord de cette espèce de mélancolie, que les Arabes appellent chatrap (en fait le kutubuth): «A cause de cette petite beste qu'ils appellent ainsi, laquelle sans repos va \& vient dessus les eaux. Les latins nomment ce petit animal entaillé, tipule: comme quelques uns escrivent, il habite ordinairement les lieux marécageux.»C'est cette maladie que Sauvages appelle melancholia errabunda. Wier cite aussi Hérodote et les Neures, peuple de Scythie; cf. aussi Wier 1885, t. II, 262ss.

56 James $1746-1748$. 
forme; la blancheur de ses poils est la même, la même la violence de ses traits; les mêmes yeux brillent; et l'image de la férocité est la même. ${ }^{57}$

Forma, vultus, imago ... On a là trois façons de dire la forme. Au XIX $\mathrm{X}^{\mathrm{e}}$ siècle, on parle encore de la lycanthropie. Calmeil écrit:

Les lycanthropes étaient quelquefois dans un état assez semblable à l'état extatique lorsque leur cerveau enfantait les hallucinations [...]. Ils n'en affirmaient pas moins, après avoir recouvré l'activité des sens, qu'ils n'avaient point rêvé, qu'ils avaient parcouru les montagnes et les précipices pour y relancer leur proie, qu'ils se sentaient harassés de fatigue; on reconnaît, même à ces derniers caractères, un état maladif bien positif. Du moment où l'activité intellectuelle s'exerce sur de pareils phénomènes sensitifs, sur de semblables idées, c'est qu'il est survenu quelque vice, quelque lésion fâcheuse dans l'entendement. ${ }^{58}$

C'est Jean Wier qui a raison et nous ramène à ce que nous ne quittons jamais, la poésie, et à l'Antiquité fabuleuse et fabulatrice (fabulosa comme dit Willis). C'est une question de poétique, de philosophie, comme de médecine. De philosophie d'abord, car il s'agit bien d'un problème de définition, de connaissance du moi. Il est intéressant de voir Locke suivre et noter les Willis' Lectures avant de devenir, comme le dit Dewhurst, le disciple de Sydenham, en s'éloignant de l'influence de Willis. Au fond, Locke se détache de la force de l'imagination, de la phantasia, de la matérialité de cette phantasia, pour définir la folie comme une erreur de jugement. La question difficile, comme le montrent les notes de Locke que publie Dewhurst, est, bien entendu, ce qu'on pourrait appeler la fixation de l'idée. Un malade peut être fou sur un point et raisonner parfaitement à côté. Question posée depuis Rufus, Arétée de Cappadoce et autres. Question en effet fondamentale. Pinel la repose à sa façon, comme nous l'avons montré59.

57 Cf. Ovide, Métamorphoses, I, 232ss:

Territus ipse fugit nactusque silentia ruri

Exululat frustraque loqui conatur; ab ipso

Colligit os rabiem solitaeque cupidine caedis

Utitur in pecudes et nunc quoque sanguine gaudet.

In villos abeunt vestes, in crura lacerti.

Fit lupus et veteris servat vestigia formae.

Canities eadem est, eadem violentia vultus,

Idem oculi lucent, eadem feritatis imago est.

On pourrait penser qu'Ovide se souvient du berger Moeris décrit par Virgile dans les Bucoliques: «Ces herbes, ces poisons cueillis dans le Pont, Moeris en personne me les a donnés (il en vient beaucoup dans le Pont); j'ai vu Moeris souvent grâce à eux se changer en loup (lupum fieri) et s'enfoncer dans le bois, souvent évoquer les âmes du fond des tombeaux et transporter dans un autre champ des moissons sur pied», Virgile, Les Bucoliques, VIII, v. 95 ss.

58 Calmeil 1845, t. I, 87. «Le juge Boguet se vantait, selon Voltaire, d'avoir fait périr plus de 600 lycanthropes ou démonolâtres»; ibid., 311.

59 Pigeaud 2001. 
Une autre question est celle de la nature de l'imagination. Que signifie le mot de phantasia? S'agit-il d'une image (apparition), s'agit-il d'une faculté? Le terme de phantasy, par exemple chez Burton, est parfois embarrassant, l'ambiguïté pouvant d'ailleurs servir.

Apparaît, à partir du I ${ }^{\mathrm{er}}$ siècle après J.-C., la question de la force intrinsèque, de la violence de l'image, qui peut être créatrice. Il existe, et pas seulement chez des philosophes, une résistance à cette force autonome de l'image. Par exemple, pour le grand médecin Bellini (1643-1704), la lycanthropie, comme d'ailleurs la cynanthropie ou la galéanthropie, naissent de l'opinio, c'est-à-dire du jugement. Sa remarque est très importante, concernant aussi le sens de la mimésis.

La lycanthropie, qu'on dit la forme première de la manie, n'est rien d'autre que la fausse imagination [lycanthropia nihil aliud est quam falsa imaginatio] qu'on est, à partir d'un homme, devenu loup. De cette imagination stupide et délirante il se produit que ce lycanthrope délirant met en branle de façon très efficace, et très habile ses esprits pour imiter les mœurs du loup [...]; il n'y a donc dans la lycanthropie aucune affinité avec une fixation du sang, aucun mouvement perturbé des esprits, quand ceux-là se meuvent de manière très étroite sous la forme supposée du loup, forme qui est activée non pas par une nature particulière des esprits, mais par l'opinion du lycanthrope. ${ }^{60}$

Malgré un latin plutôt mauvais, on ne saurait être plus clair. Il n'y a pas de transformation, mais l'idée qu'on est transformé. C'est nier la réalité de la métamorphose. Même attitude chez le médecin Le Camus (1722-1772):

Nous ne citerons pas ici toutes les idées bizarres et soutenues qu'ont enfantées les vaporeux, les hypocondriaques et les mélancoliques, idées qui produisaient chez eux de vraies sensations. Plusieurs se sont imaginés qu'ils étaient de vrais loups \& des loups garous; maladie à laquelle on a donné le nom de lycanthropie ${ }^{61}$. D'autres se sont imaginés qu'ils étaient de vrais sorciers et qu'ils assistaient au sabat. Ceux-ci s'imaginaient avoir la tête de verre \& n'osaient faire le moindre mouvement $[\ldots]$; ceux-là pensaient avoir une mouche sur le nez [...]. ${ }^{62}$

Ainsi tout cela reposerait sur une erreur de jugement. Cela mène à une aporie que mettra en évidence Pinel. L'erreur de jugement ou l'association vicieuse des idées sont insuffisantes pour expliquer l'irruption de la manie. On voit bien qu'il s'agit d'une résistance à donner une place et une fonction essentielles à un homme intérieur, qu'il soit viscères ou alambic.

\section{Sens nouveau de la mimésis}

On voit bien qu'entre l'homme intérieur de Sydenham et celui de Willis existe une différence essentielle. Celui de Sydenham n'a aucune épaisseur. Nous

60 Bellini 1717, 506s. La première édition date de 1683.

61 Il renvoie à Pline, Histoire naturelle, 8, 23.

62 Le Camus 1769, 51. 
avons utilisé l'expression de recto/verso. La notion de coextensivité de l'homme intérieur chez Willis change tout. Elle donne la possibilité d'un espace. L'homme intérieur peut se ratatiner, s'éloigner de l'homme extérieur, se déformer, prendre de la force, s'imposer, faire irruption, prendre des formes multiples, réaliser des métamorphoses. Willis parle sans cesse de métamorphoses. A la fin de son chapitre sur la melancholia ${ }^{63}$, il cite les «métamorphoses imaginaires des mélancoliques», étendant le concept, si l'on peut dire, de la lycanthropie, aux «fortunas, aut corpora sua metamorphoses imaginarias $[\ldots] \gg^{64}$.

Une fois que l'âme corporelle, affectée d'une mélancolie persistante, l'esprit étant aveuglé, s'est séparée complètement tant d'elle même que du corps, alors elle prend une forme ou une condition neuve, et, autant qu'elle le peut, elle se l'approprie réellement. ${ }^{65}$

Entendons «dans la réalité». Il faut donner aussi toute sa force à l'épithète novus. L'homme prend une forme ou des formes nouvelles. La métamorphose n'est pas une métaphore. Elle est réelle, évidente, patente, posant comme une énigme la question de l'identité derrière l'altération.

[...] il est devenu loup et conserve les traces de l'ancienne forme; même est le poil blanc, même la violence des traits; mêmes yeux qui brillent; même l'image de la férocité. ${ }^{66}$

Cette identité, bien évidemment, est à deux sens: identité avec soi-même et identification avec le loup. Arrivent alors le mythe et la poésie. Mais de quelle façon? On se souvient que le Problème $X X X$, du Pseudo-Aristote, explique l'instabilité de l'être mélancolique par celle de l'humeur bile noire ${ }^{67}$. Lui aussi montre le mélancolique comme lui-même et un autre. Cette disponibilité du mélancolique, être d'exception, peut le faire roi, poète ou philosophe. Cela pose la question de la mimésis, de l'imitation, mais d'une autre façon chez Aristote que chez Willis. Il s'agit de prendre une posture, d'agir comme tel ou tel, mais de manière si l'on peut dire théâtrale. C'est un jeu de rôles qui connaît, bien entendu, des ratages. Mais le mélancolique aristotélicien cherche, ou est poussé par une force, à sortir de soi, pour devenir un autre, ou pour lier deux choses entre elles par la métaphore. Ce qui fait du mélancolique un poète, un créateur.

63 Willis $1676,254$.

64 Par exemple en prince ou en mendiant, le malade pense et agit: Willis 1676, 254.

65 Willis 1676, 252ss. Au sujet de la melancholia specialis, Willis dit qu'il est impossible d'énumérer tous les cas: ibid., 253.

66 Fit lupus et veteris servat vestigia formae. Canities eadem est, eadem violentia vultus, Idem oculi lucent, eadem feritatis imago est. Willis 1676, 253.

67 Cf. Aristote 1988. 
Dans le cas de la métamorphose, on peut parler de la «réalité» du phantasme et de la négation de la métaphore. La mimésis de Willis remplace ici le transfert du mélancolique qui comble un espace qu'il crée d'ailleurs luimême. L'imitation de Willis remplace la métaphore. Ou plutôt les deux se combinent dans ce changement sur place qu'est la métamorphose. Le patient ne saurait faire de métaphore, car il est lui-même la métaphore. La métamorphose résout l'identité et la différence. Le même est reconnaissable sous une apparence toute autre; aliénation et altération. Imitation veut dire ici transformation, elle n'aboutit à aucune création sinon celle d'un pauvre individu qui, dans les cas extrêmes, ne fait que s'exclure de l'humanité. Il ne faut donc pas donner à la métamorphose un sens métaphorique. Comme le dit Ovide, Lycaon s'approprie, autant qu'il le peut, réellement, l'être du loup.

Chercher l'être et la vérité sous les apparences. Derrière les formes les plus diverses, unité de la mélancolie. La retrouver,l'identifier,la démasquer. Chose terrible même pour soi; surtout pour soi. Il ne faut pas, sans aucun doute, oublier la souffrance, la terrible souffrance physique et morale, de n'être rien. La totale dévalorisation de l'ego. L'immonde plasticité de soi-même. Je n'oublie pas que la métamorphose passionne en cette époque de la pensée baroque. Et, du point de vue qui nous intéresse, avec son élégance coutumière, Thomas Browne parle, comme en passant, de la «métamorphose mélancolique» de Nabuchodonosor ${ }^{68}$, lui qui marcha sept ans à quatre pattes et se prit pour un bœuf ${ }^{69}$. La question de la vérité «vraie» n'intéresse pas le malade. Où se trouve la vérité de cette souffrance? Il semble bien que la vérité, comme telle, n'intéresse pas l'hypocondriaque, ou tout au moins, qu'elle ne l'intéresse pas directement. La vérité de sa maladie est ailleurs, comme le montre Blondel ${ }^{70}$. Lorsque l'examen clinique met en évidence des lésions, le malade ne s'y intéresse pas. Il ne s'en sert pas pour alimenter sa plainte.

Poésie et hypocondrie. Nous avons vu Willis dire qu'il n'est aucune forme qui n'ait été décrite par les poètes, et la jolie formule avec laquelle, par avance, Jean Wier lui renvoyait la balle: «Ainsi que souventes fois les foles inventions des poètes ont accoutumé d'estre apuiées sur les causes naturelles [...].» Est-ce que le mythe mime la maladie? Ou est-ce que les maladies miment les mythes? Est-ce que le poète sanctionne ou propose? De toutes façons, avec la métamorphose, on sent bien que, du point de vue du malade, c'est un échec de la création. Conversion n'est pas création.

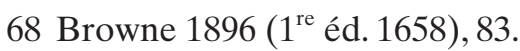

69 Le médecin Junker, qui rapproche l'hydrophobie de la lycanthropie, de la tarantule ou du tarentisme, ajoute qu'il faut diviser la lycanthropie en physique et théologique, quand le loup est dit obsédé par le diable, Stahl et Junker 1734, 930.

70 Blondel 1914, 169. 
Pour finir, revenons à Platon que citait Black à propos de la Lycanthropie. Il s'agit de République VIII 565 et du tyran qui devient loup. Platon fait d'ailleurs allusion à la légende de Lycaon, que Zeus punit en le transformant en loup, pour lui avoir fait manger de la chair humaine. En réalité, Zeus ne fait que sanctionner ce qui était déjà. «Lorsqu'on a goûté des entrailles humaines», écrit Platon, «on est fatalement changé en loup.» ${ }^{71}$ N'as-tu pas entendu conter cette histoire? Si.

De même quand le chef du peuple [...] ne sait point s'abstenir du sang des hommes de sa tribu, [...] quand, par des accusations calomnieuses [...], il les traîne devant les tribunaux et souille sa conscience en leur faisant ôter leur vie, qu'il goûte d'une langue et d'une bouche impie le sang de ses parents [...], n'est-ce pas [...] pour un tel homme une nécessité et comme une loi du destin ou de périr de la main de ses ennemis, ou de devenir tyran et d'être changé en loup? C'est une nécessité fatale. ${ }^{72}$

Des loups comme ça, nous en connaissons tous. Heureusement tous les hypocondriaques, ou quel que soit leur nom, ne deviennent pas des loups. Ils se contentent souvent d'être des singes. On peut bien appeler cette maladie protéiforme hystérie, hypocondrie, et pourquoi pas mélancolie ${ }^{73}$ ? Hypocondrie et hystérie, Stahl non plus n'y voit pas de différences essentielles. Boissier attribue la cause de cette maladie à l'amour (exclusif) de soi; pour Zimmermann, c'est la peur. Mais n'est-ce pas la même chose ${ }^{74}$ ? En réalité, c'est le petit bonhomme intérieur qui se ratatine et qui fait des grimaces. Ce n'est pas cette mélancolie-là qui fait le poète.

\section{Le Père Athanase Kircher (1602-1680)}

Je ne saurais, pour clore, résister au plaisir de citer Kircher. Ce grand homme attend encore un ouvrage d'ensemble qui rendît compte de son génie. Je ne ferai que citer ici un petit parallèle avec la singerie hypocondriaque, qui n'est pas sans intérêt. Le père Kircher s'intéresse, lui aussi, à la métamorphose qu'il

71 Hecatei fragmenta dans Fragmenta Historicorum Graecorum, 1841, fr. 375, t. 1, 31.

72 Platon, République, 565e-566a.

73 Burton préfère rattacher la lycanthropie à la manie, plutôt qu'à la mélancolie: «Le cas des filles du roi Praetus, qui se prenaient pour des vaches, était peu différent, mais leur sort était peut-être légèrement meilleur», Burton 2000, t. 1, 223. Burton cite Pline, Histoire Naturelle, $8,34,80$, qui selon lui dirait «qu'à son époque certains hommes furent métamorphosés en loups (homines in lupos verti) et reprirent ensuite leur forme». A propos des formes de la mélancolie: «Protée [...] lui-même ne possède pas autant de formes», Burton 2000, t. 1, 681.

74 «M. Donald-Monro m'a dit à Londres que son père avait fait ses études, sous Boerhaave, avec un hypochondriaque qui s'imaginait avoir les maladies que Boerhaave expliquait à chaque leçon. L'imagination de cet homme était si forte, qu'on remarquait en lui au moins quelque chose de pareil à la maladie qu'il venait d'entendre expliquer», Zimmermann 1818, t. III, 48 . 
réduit d'abord à la grimace ${ }^{75}$. On verra bien qu'il ne doit pas être un mélancolique. Il suffit à la transformation d'utiliser la déformation. On jouera alors avec des miroirs. On peut se faire un visage de rhinocéros cornu, une face de chèvre, se contempler sous la forme d'un satyre, laid, cornu, rugueux, riant de toute sa bouche ouverte, le visage rouge en plus et incendié de l'ivrogne si l'on sait glisser une feuille rouge. «En un mot, il n'est monstre si laid sous la forme duquel tu ne puisses te contempler dans un miroir, grâce à une invention de ce genre.» C'est-à-dire le mélange de quelques miroirs plans et courbes. Voulez-vous quatre yeux, deux têtes, c'est votre affaire.

Apprivoisons donc le monstrueux dans le miroir: le protéisme devient trucage, et le trucage devient un jeu que Kircher décrit avec jubilation. Mais qu'on ne s'y trompe pas. Si cette transformation extérieure est jubilatoire, il connaît bien une autre métamorphose, interne celle-là, et plus inquiétante:

Nous avons dit [écrit-il] que l'homme peut se métamorphoser [transmutari] en n'importe quelle forme. Il te faut donc savoir qu'il existe des substances naturelles qui, dès qu'elles sont absorbées par la bouche, exerçant leur pouvoir sur l'imagination [imperium in phantasiam exercentes], transforment l'homme en ce vers quoi il inclinait par-dessus tout. Ainsi je connais une racine qui, dès qu'elle a été ingérée, transforme en oies ou en canards, non pas selon l'aspect extérieur, mais l'aspect intérieur, de sorte que ceux qui l'ont ingérée se pensent vraiment des oies. Il est d'autres substances qui, une fois absorbées, transforment et transmutent en chats, chiens, loups, selon le même procédé. Mais comme ces choses sortent des limites de notre art, et qu'elles sont telles qu'en raison de beaucoup de maux, qui d'elles peuvent émerger, elles ne doivent ni ne peuvent être publiées, tout en vénérant en elles cette très grande et admirable majesté de la nature, consacrons-les à un total et éternel silence. ${ }^{76}$

\section{Bibliographie}

Aristote, L'homme de génie et la mélancolie, Problème $X X X, 1$, traduction, commentaire et notes de Jackie Pigeaud (Paris 1988)

Bellini, Lorenzo, De Urinis et pulsibus, de missione sanguinis, de febribus, de morbis capitis et pectoris, opus Laurentii Bellini, cum praefatione Hermanni Boerhaave (Leyde 1717)

Black, W., Esquisse d'une histoire de la médecine et de la chirurgie depuis leur commencement jusqu'à nos jours, ainsi que de leurs principaux auteurs, progrès, imperfections et erreurs, traduite de l'anglais par Coray (Paris 1798)

Blondel, Charles, La conscience morbide (Paris 1914)

Boissier de Sauvages, Nosologia methodica sistens morborum classes, ed. ultima (Amsterdam 1768)

- Nosologie méthodique ou distribution des maladies en classes, en genres et en espèces, suivant l'esprit de Sydenham \& la méthode des botanistes, traduite par M. Gouvion, t. 7 (Lyon 1772)

Browne, Thomas, Cyrus's Garden, or the Quincunx, ed. W. A. Greenhill (London 1896)

Burton, Robert, Anatomie de la mélancolie, traduction de Bernard Hopffner, préface de Jean Starobinski, postface de Jackie Pigeaud (Paris 2000)

Cabanis, Pierre Jean George, «Les Rapports du Physique et du Moral», dans: Euvres philosophiques, par C. Lehec et J. Cazeneuve (Paris 1956)

75 «Monstrosam faciem hominis in varia animalia transformare», Kircher 1680, Experimentum XXVII, Metamorphoseon XXVII, 130.

76 Kircher 1680, 131. 
Calmeil, Louis Florentin, article «Lycanthropie», dans: Dechambre,A., Dictionnaire des Sciences Médicales, t. 55 (Paris 1864-1889) 359ss.

- De la folie considérée sous le point de vue pathologique, philosophique, historique et judiciaire, 2 vols (Paris 1845)

Céard, Jean, La nature et les prodiges, L'insolite en France au XVI siècle (Genève 1977)

Dewhurst, Kenneth, Dr. Thomas Sydenham (1624-1689). His Life and Original Writings (London 1966)

Fragmenta Historicorum Graecorum, éd. T. Muller (Paris 1841-1870)

Freind, John, Histoire de la médecine depuis Galien jusqu'au XVI siècle (Paris 1728)

Galien, Euvres anatomiques, physiologiques et médicales, traduction de Charles Daremberg (Paris 1854-1856)

Jaeger, Werner, Diokles von Karystos (Berlin 1938)

James, Robert, Dictionnaire universel de Médecine, de Chymie, de Botanique, d'Anatomie, d'Histoire naturelle, trad. de l'anglais par Mrs Diderot, Eidous et Toussaint, 6 vols (Paris 1746-1748)

Kant, Emmanuel, Essai sur les maladies de la tête, traduction de Monique David-Ménard (Paris 1990)

Kircher, Athanase, Physiologia Kircheriana (Amstelodami 1680)

Le Camus, Antoine, Médecine de l'esprit (Paris 1769)

Le Pois, Charles, Caroli Pisonis Selectiorum Observationum et Consiliorum ... Liber singularis, cum Praefatione Hermanni Boerhaavi ... (Leyde 1733)

Longin, Du Sublime, traduction, présentation et notes de Jackie Pigeaud (Paris 1993)

Lorry, A. C., De Melancholia et morbis melancholicis (Paris 1765)

Oribase, Euvres d'Oribase, texte et traduction par Bussemaker et Daremberg, t. V (Paris 1851)

Pigeaud, Jackie, L'art et le vivant (Paris 1995)

- Poésie du corps (Paris 1999)

- Aux portes de la psychiatrie. Pinel, l'Ancien et le Moderne (Paris 2001)

Stahl, Georg Ernst, Conspectus Medicinae theoretico-practicae tabulis CXXXVIII omnes primarios morbos, methodo Stahliana tractandos ... auctore D. Joanne Junckero (Halle 1734)

- «Dissertatio de motu tonico», dans: Theoria medica vera, physiologicam et pathologiam, 2 vols (Halle 1708)

Sydenham, Thomas, Opera medica (Genève 1716)

- Opera universa, Editio novissima (Leyde 1726)

- Euvres de médecine pratique de Thomas Sydenham, médecin du dix-septième siècle et célèbre praticien de Londres, traduites en français sur la dernière édition anglaise par A. F. Jault (Paris 1776)

Veith, Ilza, Hysteria, the History of a Disease (Chicago/London 1965)

Virgile, Bucoliques, texte établi et traduit par E. De Saint-Denis (Paris 1967)

Whytt, Robert, Traité des maladies nerveuses, hypocondriaques et hystériques (Paris 1777)

Wier, Jean, Histoires, disputes et discours des illusions et impostures des diables et magiciens infâmes ... (Paris 1885)

Willis, Thomas, De anima brutorum, quae hominis vitalis ac sensitiva est. Pars secunda pathologica sive De Morbis qui ad animam corpoream, ejusque subjecta, scilicet cerebrum \& genus nervosum spectant (Lugduni 1676)

- Thomas Willis's Oxford Lectures, ed. K. Dewhurst (Oxford/Stanford 1980)

Zimmermann, Georg, Traité de l'expérience en général, et en particulier dans l'art de guérir, t. III (Montpellier 1818) 